\title{
Protective Agent
}

National Cancer Institute

\section{Source}

National Cancer Institute. Protective Agent. NCI Thesaurus. Code C26170.

A class of agents used in prophylactic or curative therapy to attenuate the negative side effects of toxins or drugs. Protective agents belong to various chemical classes, acting through various pharmacological mechanisms. They may neutralize exogenous poisons, coat mucosa for protection against physical or chemical damage, offer antioxidant protection against free radicals or ionizing radiation, or induce endogenous detoxifying enzymes. ( $\mathrm{NCl04)}$ 